\title{
Structural improvement of new thiazolidinones compounds with antinociceptive activity in experimental chemotherapy-induced painful neuropathy
}

\author{
Diogo Rodrigo Magalhaes Moreira ${ }^{1}$ | Dourivaldo Silva Santos ${ }^{2}$ | \\ Renan Fernandes do Espírito Santo ${ }^{1,2}$ | Flávia Evangelista dos Santos ${ }^{2}$ | \\ Gevanio Bezerra de Oliveira Filho $^{3}$ | Ana Cristina Lima Leite ${ }^{3}$ | \\ Milena Botelho Pereira Soares $^{1,4}$ | Cristiane Flora Villarreal ${ }^{1,2}$ (D)
}

${ }^{1}$ Centro de Pesquisas Gonçalo

Moniz, FIOCRUZ, Salvador, Bahia, Brazil

${ }^{2}$ Faculdade de Farmácia, Universidade

Federal da Bahia, Salvador, Bahia, Brazil

${ }^{3}$ Departamento de Ciências

Farmacêuticas, Centro de Ciências

da Saúde, Universidade Federal de

Pernambuco, Recife, PE, Brazil

${ }^{4}$ Centro de Biotecnologia e Terapia

Celular, Hospital São Rafael, Salvador,

Bahia, Brazil

\section{Correspondence}

Cristiane Flora Villarreal, Laboratório de

Engenharia Tecidual e Imunofarmacologia,

Centro de Pesquisa Gonçalo Moniz,

Fundação Oswaldo Cruz, Salvador, BA,

Brazil.

Email: cfv@ufba.br

Funding information

FAPESB, Grant/Award Number: PNX

0009/2009 and RED0010/2012.
Chemotherapy-induced neuropathy is a disabling pain condition resulting from chemotherapy for cancers. Up to now, no drug is available to cure chemotherapyinduced neuropathy. In the present study, we describe the structural design, synthesis, chemical and pharmacological characterization of 15 thiazolidinones, a class of potential analgesic compounds. The synthesis of new thiazolidinones was achieved by using the thiazolidinone heterocyclic as main structural pharmacophoric group and varying the substituents attached to the phenyl near to the iminic bond. The analgesic potential of the compounds was investigated in a mice model of oxaliplatininduced neuropathic pain, using von Frey, rota-rod and open-field tests. Except for compound 14, these thiazolidinones exhibited antinociceptive property without causing motor impairment. Thiazolidinones 12, 15 and 16 displayed a dose-dependent antinociceptive effect, with similar efficacy and enhanced potency than gabapentin, the gold standard drug used for neuropathic pain. In addition, the antinociceptive activity of $\mathbf{1 6}$ lasted longer than gabapentin. The antinociceptive effect of thiazolidinones was prevented by GW9662, a PPAR $\gamma$ antagonist. The main antinociceptive compounds exhibited positive Lipinski's index, predicting their oral bioavailability. In conclusion, the structural design performed here led to the identification of new compounds endowed with potent antinociceptive activity, potentially useful to treat chemotherapy-induced neuropathic pain.

\section{K E Y W O R D S}

analgesic, glitazones, neuropathic pain, oxaliplatin, PPAR

\section{INTRODUCTION}

The neuropathic pain is mainly initiated by a primary lesion in the nervous system, commonly caused by a trauma, infection or ischemia. Alternatively, a dysfunction can be induced by cancer and the use of chemotherapy. ${ }^{[1]}$ This chronic syndrome is characterized by spontaneous pain and an increased response to painful and innocuous stimuli. ${ }^{[2]}$ Despite the great morbidity, social cost and negative impact on quality of life, the neuropathic pain has limited therapeutic options, due to the reduced number of pharmaceutical with efficacy in this syndrome ${ }^{[3]}$ Painful peripheral neuropathy is a common and dose-limiting side effect of anticancer drugs, such as vinca alkaloids, taxanes, epothilones and platinum-based 
compounds. ${ }^{[4,5]}$ Due to this chemotherapy-induced neuropathy, many oncological patients are unable to receive the entire treatment. ${ }^{[6]}$ The prevalence of chemotherapy-induced neuropathy varies from $10 \%$ to $80 \%$, according to the drug used. For instance, the platinum drug oxaliplatin, a wellknown peripheral neuropathy inducer, leads to the developing pain signs in $75 \%$ of the patients. ${ }^{[7]} \mathrm{Up}$ to now, no drug is available to reliably prevent or cure oxaliplatin-induced neuropathy, stressing the importance of the development of new agents with analgesic activity during neuropathic conditions. ${ }^{[8]}$

Thiazolidinones are small molecules, most known as antidiabetic glitazones, that act as agonists of nuclear receptors, mainly the peroxisome proliferator-activated receptors (PPAR) family. ${ }^{[9-11]}$ Thiazolidinones are considered pleiotropic agents with hypoglycemic, antihyperlipidemic, antiinflammatory and antinociceptive properties. ${ }^{[10,12-15]}$ Park and collaborators first described the neuroprotective properties of thiazolidinones, showing that pretreatment with pioglitazone prevented numerous consequences of spinal cord injury, including neuronal damage, inflammation and behavioral neuropathic pain. ${ }^{[16]}$ After this original work, it is becoming increasingly clear that the use of thiazolidinones, such as rosiglitazone and pioglitazone, represents a promising therapeutic strategy for neuropathic pain. ${ }^{[17-19]}$ The oxaliplatin-induced mechanisms causing the neuropathic pain are still unclear, but a relationship between chemotherapydependent mitochondrial alteration and neuropathy has been established. Recently, it was demonstrated a protective role of PPAR signaling in oxaliplatin neurotoxicity. ${ }^{[20]}$ Therefore, the development of novel PPAR agonist-thiazolidinones endowed with analgesic property in chemotherapy-induced neuropathic pain holds great potential. In the present study, a series of thiazolidinone compounds were structurally planned from aryl thiosemicarbazones, prepared and chemically characterized by spectroscopy analyses. Following this, the analgesic potential of these new thiazolidinone compounds was evaluated in a mouse model of oxaliplatin-induced neuropathic pain.

\section{2 | MATERIALS AND METHODS}

\section{1 | Reagents and spectra analysis}

All reagents were used as purchased from commercial sources (Sigma-Aldrich, Acros Organics, Vetec or Fluka). Progress of the reactions was followed by thin-layer chromatography (silica gel 60 F254 in aluminum foil). IR was determined in $\mathrm{KBr}$ pellets. For NMR, it was used a Bruker AMX-300 MHz (300 MHz for ${ }^{1} \mathrm{H}$ and $75.5 \mathrm{MHz}$ for ${ }^{13} \mathrm{C}$ ) instrument. DMSO- $d_{6}$ was purchased from CIL. Chemical shifts are reported in ppm, and multiplicities are given as $\mathrm{s}$ (singlet), d (doublet), $\mathrm{t}$ (triplet), q (quartet), $\mathrm{m}$ (multiplet) and dd (doublet of doublets) and coupling constants $(J)$ in hertz. NH signals were localized in each spectrum after the addition of a few drops of $\mathrm{D}_{2} \mathrm{O}$. Structural assignments were corroborated by DEPT analysis. Mass spectrometry experiments were performed on a Q-TOF spectrometer LC-IT-TOF (Shimadzu). When otherwise specified, ESI was carried out in the positive ion mode. Reactions in an ultrasound bath were carried out under frequency of $40 \mathrm{kHz}(180 \mathrm{~W})$ and without external heating.

\section{2 | Synthesis of aryl thiosemicarbazones (2)}

Compounds were prepared as described in the literature. ${ }^{[21]}$ These compounds were isolated as solid crystals and recrystallized in ethanol prior to use.

\section{3 | Synthesis of aryl thiazolidinones (3-17)}

Example for compound (3): aryl thiosemicarbazone (5 mmol, $1.1 \mathrm{~g})$ and $15 \mathrm{mmol}(1.2 \mathrm{~g})$ of anhydrous sodium acetate were solubilized in $100 \mathrm{ml}$ of ethanol and were added to a $250-\mathrm{ml}$ round-bottomed flask under magnetic stirring and warmed for $30 \mathrm{~min}$. Ethyl 2-chloro-2-phenyl acetate $(2.0 \mathrm{~g}, 10 \mathrm{mmol})$ was added in portions, and the reaction mixture was stirred under reflux for $10 \mathrm{hr}$. After cooling back to r.t., salt was filtered off and the solvent was evaporated for half of its volume and cooled to $0^{\circ} \mathrm{C}$ overnight. A colorless solid was obtained, filtered in Büchner funnel with sintered disc filter, washed with cold water and dried in vacuum.

\subsection{1 | 5-Phenyl-2-(2-(1-phenylethylidene) hydrazinyl)thiazol-4(5H)-one (3)}

Crystallization from toluene afforded white crystals, yield 70\%. M.p. $\left({ }^{\circ} \mathrm{C}\right): 182$. IR ( $\left.\mathrm{KBr}\right): 3,358(\mathrm{~N}-\mathrm{H}), 1,713(\mathrm{C}=\mathrm{O})$, $1,601(\mathrm{C}=\mathrm{N}) \mathrm{cm}^{-1} .{ }^{1} \mathrm{H}$ NMR $\left(300 \mathrm{MHz}, \mathrm{DMSO}-d_{6}\right): \delta 2.16$ $\left(\mathrm{CH}_{3}\right), 5.00$ (s, 1H, CH), 6.89-7.01 (m, 3H, Ar), 7.21-7.29 $(\mathrm{m}, 7 \mathrm{H}, \mathrm{Ar}), 10.80(\mathrm{~s}, 1 \mathrm{H}, \mathrm{NH}) .{ }^{13} \mathrm{C} \mathrm{NMR}(75.5 \mathrm{MHz}$, DMSO- $\left.d_{6}\right): \delta 25.1\left(\mathrm{CH}_{3}\right), 51.4(\mathrm{CH}), 114.2(\mathrm{CH}, \mathrm{Ar}), 114.2$ $(\mathrm{CH}, \mathrm{Ar}), 120.9(\mathrm{CH}, \mathrm{Ar}), 128.3(\mathrm{CH}, \mathrm{Ar}), 128.7(\mathrm{CH}$, Ar), $129.4(\mathrm{CH}, \mathrm{Ar}), 130.4(\mathrm{CH}, \mathrm{Ar}), 137.4(\mathrm{C}-\mathrm{N}, \mathrm{Ar})$, $157.1(\mathrm{C}=\mathrm{N}), 162.8(\mathrm{C}-\mathrm{O}), 164.4(\mathrm{~S}-\mathrm{C}=\mathrm{N}), 174.2(\mathrm{C}=\mathrm{O})$. HRESIMS: $310.0615[\mathrm{M}+\mathrm{H}]^{+}$.

\subsection{2 | 5-Phenyl-2-(2-(1-(4-tolyl)ethylidene) hydrazinyl)thiazol-4(5H)-one (4)}

Crystallization from toluene afforded white crystals, yield 63\%. M.p. $\left({ }^{\circ} \mathrm{C}\right):$ 201. IR ( $\left.\mathrm{KBr}\right): 3,350(\mathrm{~N}-\mathrm{H}), 1,709(\mathrm{C}=\mathrm{O})$, $1,598(\mathrm{C}=\mathrm{N}) \mathrm{cm}^{-1} .{ }^{1} \mathrm{H}$ NMR $\left(300 \mathrm{MHz}, \mathrm{DMSO}-d_{6}\right): \delta 2.11$ $\left(\mathrm{CH}_{3}\right), 2.30\left(\mathrm{CH}_{3}\right), 3.41$ (br s, $\left.1 \mathrm{H}, \mathrm{NH}\right), 5.00(\mathrm{~s}, 1 \mathrm{H}, \mathrm{CH})$, 6.92-7.03 (m, 3H, Ar), 7.14-7.22 (m, 6H, Ar). ${ }^{13} \mathrm{C}$ NMR $\left(75.5 \mathrm{MHz}, \mathrm{DMSO}-d_{6}\right): \delta 25.1\left(\mathrm{CH}_{3}\right), 30.9\left(\mathrm{CH}_{3}\right), 50.2$ 
(CH), $114.2(\mathrm{CH}, \mathrm{Ar}), 114.2(\mathrm{CH}, \mathrm{Ar}), 128.3(\mathrm{CH}, \mathrm{Ar}), 128.7$ $(\mathrm{CH}, \mathrm{Ar}), 130.1(\mathrm{CH}, \mathrm{Ar}), 130.4(\mathrm{CH}, \mathrm{Ar}), 137.4(\mathrm{C}-\mathrm{N}, \mathrm{Ar})$, $157.1(\mathrm{C}=\mathrm{N}), 162.8(\mathrm{C}-\mathrm{O}), 164.4(\mathrm{~S}-\mathrm{C}=\mathrm{N}), 174.2(\mathrm{C}=\mathrm{O})$. HRESIMS: $324.1321[\mathrm{M}+\mathrm{H}]^{+}$.

\subsection{3 | 5-Phenyl-2-(2-(1-(4-anisyl)ethylidene) hydrazinyl)thiazol-4(5H)-one (5)}

Crystallization from toluene afforded white crystals, yield 70\%. M.p. $\left({ }^{\circ} \mathrm{C}\right): 189$. IR $(\mathrm{KBr}): 3,352(\mathrm{~N}-\mathrm{H}), 1,710(\mathrm{C}=\mathrm{O})$, $1,601(\mathrm{C}=\mathrm{N}) \mathrm{cm}^{-1} .{ }^{1} \mathrm{H}$ NMR $\left(300 \mathrm{MHz}, \mathrm{DMSO}-d_{6}\right): \delta 2.11$ $\left(\mathrm{CH}_{3}\right), 3.41(\mathrm{br} \mathrm{s}, 1 \mathrm{H}, \mathrm{NH}), 4.30\left(\mathrm{OCH}_{3}\right), 5.00(\mathrm{~s}, 1 \mathrm{H}, \mathrm{CH})$, 6.92-7.03 (m, 3H, Ar), 7.10-7.16 (m, 6H, Ar). ${ }^{13} \mathrm{C}$ NMR $\left(75.5 \mathrm{MHz}, \mathrm{DMSO}-d_{6}\right): \delta 20.1\left(\mathrm{CH}_{3}\right), 50.9\left(\mathrm{OCH}_{3}\right), 52.2$ $(\mathrm{CH}), 114.2(\mathrm{CH}, \mathrm{Ar}), 114.2(\mathrm{CH}, \mathrm{Ar}), 120.9(\mathrm{CH}, \mathrm{Ar})$, $128.3(\mathrm{CH}, \mathrm{Ar}), 128.7$ (CH, Ar), $130.1(\mathrm{CH}, \mathrm{Ar}), 130.4(\mathrm{CH}$, Ar), $137.4(\mathrm{C}-\mathrm{N}, \mathrm{Ar}), 157.1(\mathrm{C}=\mathrm{N}), 162.8(\mathrm{C}-\mathrm{O}), 164.4$ $(\mathrm{S}-\mathrm{C}=\mathrm{N}), 176.9(\mathrm{C}=\mathrm{O})$. HRESIMS: $340.0950[\mathrm{M}+\mathrm{H}]^{+}$.

\subsection{4 | 5-Phenyl-2-(2-(1-(4-tertbutylphenyl) ethylidene)hydrazinyl)thiazol-4(5H)-one (6)}

Crystallization from toluene afforded white crystals, yield 55\%. M.p. $\left({ }^{\circ} \mathrm{C}\right): 162$. IR $(\mathrm{KBr}): 3,330(\mathrm{~N}-\mathrm{H}), 1,710(\mathrm{C}=\mathrm{O})$, $1,600(\mathrm{C}=\mathrm{N}) \mathrm{cm}^{-1} .{ }^{1} \mathrm{H}$ NMR $\left(300 \mathrm{MHz}, \mathrm{DMSO}-d_{6}\right): \delta 2.03$ (br s, $\left.9 \mathrm{H}, \mathrm{CH}_{3}\right), 2.11\left(\mathrm{CH}_{3}\right), 3.41($ br s, $1 \mathrm{H}, \mathrm{NH}), 4.97$ (s, $1 \mathrm{H}, \mathrm{CH}), 6.87-7.00$ (m, 3H, Ar), 7.11-7.18 (m, 6H, Ar). ${ }^{13} \mathrm{C}$ NMR (75.5 MHz, DMSO- $\left.d_{6}\right): \delta 18.2\left(\mathrm{CH}_{3}\right), 20.1\left(\mathrm{CH}_{3}\right), 36.2$ (C), $52.2(\mathrm{CH}), 114.2(\mathrm{CH}, \mathrm{Ar}), 114.2(\mathrm{CH}, \mathrm{Ar}), 120.9(\mathrm{CH}$, $\mathrm{Ar}), 128.3(\mathrm{CH}, \mathrm{Ar}), 128.7$ (CH, Ar), $130.1(\mathrm{CH}, \mathrm{Ar}), 130.4$ $(\mathrm{CH}, \mathrm{Ar}), 137.4(\mathrm{C}-\mathrm{N}, \mathrm{Ar}), 157.1(\mathrm{C}=\mathrm{N}), 162.8(\mathrm{C}-\mathrm{O}), 165.2$ $(\mathrm{S}-\mathrm{C}=\mathrm{N}), 176.9(\mathrm{C}=\mathrm{O})$. HRESIMS: $365.1732[\mathrm{M}+\mathrm{H}]^{+}$.

\subsection{5 | 5-Phenyl-2-(2-(1-(4-1',1-biphenyl) ethylidene)hydrazinyl)thiazol-4(5H)-one (7)}

Crystallization from toluene afforded white crystals, yield 53\%. M.p. $\left({ }^{\circ} \mathrm{C}\right):$ 187. IR (KBr): 3,343 (N-H), 1,708 $(\mathrm{C}=\mathrm{O})$, $1,608(\mathrm{C}=\mathrm{N}) \mathrm{cm}^{-1} .{ }^{1} \mathrm{H}$ NMR $\left(300 \mathrm{MHz}, \mathrm{DMSO}-d_{6}\right): \delta 2.12$ $\left(\mathrm{CH}_{3}\right), 3.36$ (br s, $\left.1 \mathrm{H}, \mathrm{NH}\right), 5.05(\mathrm{~s}, 1 \mathrm{H}, \mathrm{CH}), 6.87-7.00(\mathrm{~m}$, $3 \mathrm{H}, \mathrm{Ar}), 7.43$ (t, 2H, Ar), 7.49-7.54 (m, 6H, Ar), 7.62 (d, 2 $\mathrm{H}, \mathrm{Ar}) .{ }^{13} \mathrm{C}$ NMR $\left(75.5 \mathrm{MHz}, \mathrm{DMSO}-d_{6}\right): \delta 18.2\left(\mathrm{CH}_{3}\right), 20.1$ $\left(\mathrm{CH}_{3}\right), 36.2(\mathrm{C}), 52.2(\mathrm{CH}), 114.2(\mathrm{CH}, \mathrm{Ar}), 114.2(\mathrm{CH}, \mathrm{Ar})$, 114.9 (CH, Ar), 120.5 (C, Ar), $120.9(\mathrm{CH}, \mathrm{Ar}), 129.3(\mathrm{CH}$, $\mathrm{Ar}), 129.2$ (CH, Ar), 130.1 (CH, Ar), 130.4 (C, Ar), 138.4 $(\mathrm{C}-\mathrm{N}, \operatorname{Ar}), 157.1(\mathrm{C}=\mathrm{N}), 162.8(\mathrm{C}-\mathrm{O}), 165.2(\mathrm{~S}-\mathrm{C}=\mathrm{N})$, $176.9(\mathrm{C}=\mathrm{O})$. HRESIMS: $386.1247[\mathrm{M}+\mathrm{H}]^{+}$.

\subsection{6 | 5-Phenyl-2-(2-(1-(4-hydroxyphenyl) ethylidene)hydrazinyl)thiazol-4(5H)-one (8)}

Crystallization from toluene afforded white crystals, yield 76\%. M.p. $\left({ }^{\circ} \mathrm{C}\right): 203$ IR $(\mathrm{KBr}): 3,240(\mathrm{~N}-\mathrm{H})$,
1,711 $(\mathrm{C}=\mathrm{O}), 1,597(\mathrm{C}=\mathrm{N}) \mathrm{cm}^{-1} \cdot{ }^{1} \mathrm{H}$ NMR $(300 \mathrm{MHz}$, DMSO- $\left.d_{6}\right): \delta 2.06\left(\mathrm{CH}_{3}\right), 3.30($ br s, $1 \mathrm{H}, \mathrm{NH}), 5.01(\mathrm{~s}$, $1 \mathrm{H}, \mathrm{CH}), 6.92-7.03(\mathrm{~m}, 3 \mathrm{H}, \mathrm{Ar}), 7.10-7.16(\mathrm{~m}, 6 \mathrm{H}, \mathrm{Ar})$, $7.42(\mathrm{t}, 2 \mathrm{H}, \mathrm{Ar}) .{ }^{13} \mathrm{C}$ NMR $\left(75.5 \mathrm{MHz}, \mathrm{DMSO}-d_{6}\right): \delta 20.1$ $\left(\mathrm{CH}_{3}\right), 50.7(\mathrm{CH}), 113.9(\mathrm{CH}, \mathrm{Ar}), 114.2(\mathrm{CH}, \mathrm{Ar}), 120.9$ $(\mathrm{CH}, \mathrm{Ar}), 128.3(\mathrm{CH}, \mathrm{Ar}), 128.7(\mathrm{CH}, \mathrm{Ar}), 130.1(\mathrm{CH}$, Ar), $132.0(\mathrm{CH}, \mathrm{Ar}), 137.4(\mathrm{C}-\mathrm{N}, \mathrm{Ar}), 157.1(\mathrm{C}=\mathrm{N})$, $162.8(\mathrm{C}-\mathrm{O}), 164.4(\mathrm{~S}-\mathrm{C}=\mathrm{N}), 175.0(\mathrm{C}=\mathrm{O})$. HRESIMS: $326.1034[\mathrm{M}+\mathrm{H}]^{+}$.

\subsection{7 | 5-Phenyl-2-(2-(1-(4-nitrophenyl) ethylidene)hydrazinyl)thiazol-4(5H)-one (9)}

Crystallization from toluene afforded yellowish crystals, yield 55\%. M.p. $\left({ }^{\circ} \mathrm{C}\right): 211-212$ IR $(\mathrm{KBr}): 3,311(\mathrm{~N}-\mathrm{H})$, $1,710(\mathrm{C}=\mathrm{O}), 1,597(\mathrm{C}=\mathrm{N}) \mathrm{cm}^{-1} \cdot{ }^{1} \mathrm{H}$ NMR $(300 \mathrm{MHz}$, DMSO- $\left.d_{6}\right): \delta 2.06\left(\mathrm{CH}_{3}\right), 5.01(\mathrm{~s}, 1 \mathrm{H}, \mathrm{CH}), 6.92-7.03(\mathrm{~m}$, $3 \mathrm{H}, \mathrm{Ar}), 7.10-7.16$ (m, 6H, Ar), 7.52 (t, 2H, Ar). ${ }^{13} \mathrm{C} \mathrm{NMR}$ $\left(75.5 \mathrm{MHz}, \mathrm{DMSO}-d_{6}\right): \delta 20.1\left(\mathrm{CH}_{3}\right), 50.7(\mathrm{CH}), 113.9(\mathrm{CH}$, Ar), 114.0 (CH, Ar), 120.9 (CH, Ar), $128.3(\mathrm{CH}, \mathrm{Ar}), 128.7$ $(\mathrm{CH}, \mathrm{Ar}), 130.1(\mathrm{CH}, \mathrm{Ar}), 132.0(\mathrm{CH}, \mathrm{Ar}), 135.0(\mathrm{C}-\mathrm{N}, \mathrm{Ar})$, $157.1(\mathrm{C}=\mathrm{N}), 162.8(\mathrm{C}-\mathrm{O}), 164.4(\mathrm{~S}-\mathrm{C}=\mathrm{N}), 175.0(\mathrm{C}=\mathrm{O})$. HRESIMS: $354.9743[\mathrm{M}+\mathrm{H}]^{+}$.

\subsection{8 | 5-Phenyl-2-(2-(1-(4-fluorphenyl) ethylidene)hydrazinyl)thiazol-4(5H)-one (10)}

Crystallization from toluene afforded white crystals, yield 73\%. M.p. $\left({ }^{\circ} \mathrm{C}\right)$ : 201 IR (KBr): 3,311 (N-H), $1,710(\mathrm{C}=\mathrm{O}), 1,605(\mathrm{C}=\mathrm{N}) \mathrm{cm}^{-1} .{ }^{1} \mathrm{H}$ NMR $(300 \mathrm{MHz}$, DMSO- $\left.d_{6}\right): \delta 2.06\left(\mathrm{CH}_{3}\right), 5.21(\mathrm{~s}, 1 \mathrm{H}, \mathrm{CH}), 6.90-6.97(\mathrm{~m}$, $3 \mathrm{H}, \mathrm{Ar}), 7.10-7.16(\mathrm{~m}, 6 \mathrm{H}, \mathrm{Ar}), 7.52(\mathrm{t}, 2 \mathrm{H}, \mathrm{Ar}) .{ }^{13} \mathrm{C} \mathrm{NMR}$ $\left(75.5 \mathrm{MHz}, \mathrm{DMSO}-d_{6}\right): \delta 22.1\left(\mathrm{CH}_{3}\right), 50.7(\mathrm{CH}), 111.0$ $(\mathrm{C}, \mathrm{Ar}), 113.9(\mathrm{CH}, \mathrm{Ar}), 114.0(\mathrm{CH}, \mathrm{Ar}), 120.9(\mathrm{CH}, \mathrm{Ar})$, $128.3(\mathrm{CH}, \mathrm{Ar}), 128.7$ (CH, Ar), $130.1(\mathrm{CH}, \mathrm{Ar}), 131.1$ $(\mathrm{C}, \mathrm{Ar}), 132.0(\mathrm{CH}, \mathrm{Ar}), 135.0(\mathrm{C}-\mathrm{N}, \mathrm{Ar}), 157.1(\mathrm{C}=\mathrm{N})$, $162.8(\mathrm{C}-\mathrm{O}), 159.3(\mathrm{~S}-\mathrm{C}=\mathrm{N}), 175.0(\mathrm{C}=\mathrm{O})$. HRESIMS: $328.8231[\mathrm{M}+\mathrm{H}]^{+}$.

\subsection{9 | 5-Phenyl-2-(2-(1-(4-chlorophenyl) ethylidene)hydrazinyl)thiazol-4(5H)-one (11)}

Crystallization from toluene afforded white crystals, yield 73\%. M.p. $\left({ }^{\circ} \mathrm{C}\right)$ : 232 IR (KBr): 3,401 (N-H), $1,710(\mathrm{C}=\mathrm{O}), 1,605(\mathrm{C}=\mathrm{N}) \mathrm{cm}^{-1} \cdot{ }^{1} \mathrm{H}$ NMR $(300 \mathrm{MHz}$, DMSO- $\left.d_{6}\right)$ : $\delta 2.06\left(\mathrm{CH}_{3}\right), 5.00(\mathrm{~s}, 1 \mathrm{H}, \mathrm{CH}), 6.90-6.97(\mathrm{~m}$, $3 \mathrm{H}, \mathrm{Ar}), 7.10-7.16$ (m, 6H, Ar), 7.52 (t, 2H, Ar). ${ }^{13} \mathrm{C} \mathrm{NMR}$ $\left(75.5 \mathrm{MHz}\right.$, DMSO- $\left.d_{6}\right): \delta 21.1\left(\mathrm{CH}_{3}\right), 50.7(\mathrm{CH}), 111.0$ (C, Ar), 113.9 (CH, Ar), $114.0(\mathrm{CH}, \mathrm{Ar}), 120.9$ (CH, Ar), $128.3(\mathrm{CH}, \mathrm{Ar}), 129.7(\mathrm{CH}, \mathrm{Ar}), 130.1(\mathrm{CH}, \mathrm{Ar}), 131.1$ $(\mathrm{C}, \mathrm{Ar}), 132.0(\mathrm{CH}, \mathrm{Ar}), 135.0(\mathrm{C}-\mathrm{N}, \mathrm{Ar}), 157.1(\mathrm{C}=\mathrm{N})$, $162.8(\mathrm{C}-\mathrm{O}), 164.4(\mathrm{~S}-\mathrm{C}=\mathrm{N}), 175.0(\mathrm{C}=\mathrm{O})$. HRESIMS: $344.9878[\mathrm{M}+\mathrm{H}]^{+}$. 


\subsubsection{0 | 5-Phenyl-2-(2-(1-(4- trifluormethylphenyl)ethylidene)hydrazinyl) thiazol-4(5H)-one (12)}

Crystallization from toluene afforded white crystals, yield 45\%. M.p. $\left({ }^{\circ} \mathrm{C}\right): 197 \mathrm{IR}(\mathrm{KBr}): 3,310(\mathrm{~N}-\mathrm{H}), 1,710(\mathrm{C}=\mathrm{O})$, $1,605(\mathrm{C}=\mathrm{N}) \mathrm{cm}^{-1}$. ${ }^{1} \mathrm{H}$ NMR (300 MHz, DMSO- $\left.d_{6}\right): \delta 2.06$ $\left(\mathrm{CH}_{3}\right), 5.00$ (s, 1H, CH), 6.90-6.97 (m, 3H, Ar), 7.10-7.15 (m, 6H, Ar), 7.50 (t, 2H, Ar). HRESIMS: $378.0721[\mathrm{M}+\mathrm{H}]^{+}$.

\subsubsection{1 | 5-Phenyl-2-(2-(1-(3-} trifluormethylphenyl)ethylidene)hydrazinyl) thiazol-4(5H)-one (13)

Crystallization from toluene afforded white crystals, yield 41\%. M.p. $\left({ }^{\circ} \mathrm{C}\right): 201 \mathrm{IR}(\mathrm{KBr}): 3,307(\mathrm{~N}-\mathrm{H}), 1,709(\mathrm{C}=\mathrm{O})$, $1,605(\mathrm{C}=\mathrm{N}) \mathrm{cm}^{-1} \cdot{ }^{1} \mathrm{H}$ NMR $\left(300 \mathrm{MHz}, \mathrm{DMSO}-d_{6}\right): \delta$ $2.06\left(\mathrm{CH}_{3}\right), 5.00(\mathrm{~s}, 1 \mathrm{H}, \mathrm{CH}), 6.86-6.96(\mathrm{~m}, 3 \mathrm{H}, \mathrm{Ar}), 7.25-$ 7.34 (m, 6H, Ar), 7.61 (d, 2H, Ar). ${ }^{13} \mathrm{C}$ NMR $(75.5 \mathrm{MHz}$, DMSO- $\left.d_{6}\right): \delta 20.1\left(\mathrm{CH}_{3}\right), 50.7(\mathrm{CH}), 111.0(\mathrm{C}, \mathrm{Ar}), 113.9$ $(\mathrm{CH}, \mathrm{Ar}), 114.0(\mathrm{CH}, \mathrm{Ar}), 120.9(\mathrm{CH}, \mathrm{Ar}), 128.3(\mathrm{CH}, \mathrm{Ar})$, 128.7 (CH, Ar), 130.1 (CH, Ar), 131.1 (C, Ar), $132.0(\mathrm{CH}$, Ar), $135.0(\mathrm{C}-\mathrm{N}$, Ar $), 157.1(\mathrm{C}=\mathrm{N}), 162.8(\mathrm{C}-\mathrm{O}), 164.4$ $(\mathrm{S}-\mathrm{C}=\mathrm{N}), 176.8(\mathrm{C}=\mathrm{O})$. HRESIMS: $378.1211[\mathrm{M}+\mathrm{H}]^{+}$.

\subsubsection{2 | 5-Phenyl-2-(2-(1-(3,4- dimethoxyphenyl)ethylidene)hydrazinyl) thiazol-4(5H)-one (14)}

Crystallization from toluene afforded white crystals, yield 53\%. M.p. $\left({ }^{\circ} \mathrm{C}\right): 169 \mathrm{IR}(\mathrm{KBr}): 3,401(\mathrm{~N}-\mathrm{H}), 1,710(\mathrm{C}=\mathrm{O})$, $1,605(\mathrm{C}=\mathrm{N}) \mathrm{cm}^{-1} \cdot{ }^{1} \mathrm{H}$ NMR $\left(300 \mathrm{MHz}, \mathrm{DMSO}-d_{6}\right): \delta$ $2.11\left(\mathrm{CH}_{3}\right), 3.41(\mathrm{br} \mathrm{s}, 1 \mathrm{H}, \mathrm{NH}), 4.33\left(\mathrm{OCH}_{3}\right), 5.00(\mathrm{~s}, 1 \mathrm{H}$, $\mathrm{CH}), 6.90-7.03$ (m, 3H, Ar), 7.10-7.16 (m, 5H, Ar). ${ }^{13} \mathrm{C}$ NMR (75.5 MHz, DMSO- $\left.d_{6}\right)$ : $\delta 20.1\left(\mathrm{CH}_{3}\right), 52.1\left(\mathrm{OCH}_{3}\right)$, $53.0(\mathrm{CH}), 114.2(\mathrm{CH}, \mathrm{Ar}), 115.0(\mathrm{C}, \mathrm{Ar}), 120.9(\mathrm{CH}, \mathrm{Ar})$, $128.3(\mathrm{CH}, \mathrm{Ar}), 128.7(\mathrm{CH}, \mathrm{Ar}), 130.1(\mathrm{CH}, \mathrm{Ar}), 132.3$ $(\mathrm{CH}, \mathrm{Ar}), 137.4(\mathrm{C}-\mathrm{N}, \mathrm{Ar}), 157.1(\mathrm{C}=\mathrm{N}), 162.8(\mathrm{C}-\mathrm{O})$, $164.4(\mathrm{~S}-\mathrm{C}=\mathrm{N}), 176.9(\mathrm{C}=\mathrm{O})$. HRESIMS: 370.1133 $[\mathrm{M}+\mathrm{H}]^{+}$.

\subsubsection{3 | 5-Phenyl-2-(2-(1-(3,4- hydroxyphenyl)ethylidene)hydrazinyl)thiazol- 4(5H)-one (15)}

Crystallization from toluene afforded white crystals, yield 50\%. M.p. $\left({ }^{\circ} \mathrm{C}\right): 189 \mathrm{IR}(\mathrm{KBr}): 3,401(\mathrm{OH}), 1,708(\mathrm{C}=\mathrm{O})$, $1,590(\mathrm{C}=\mathrm{N}) \mathrm{cm}^{-1} \cdot{ }^{1} \mathrm{H}$ NMR $\left(300 \mathrm{MHz}, \mathrm{DMSO}-d_{6}\right): \delta$ $2.11\left(\mathrm{~s}, 1 \mathrm{H}, \mathrm{CH}_{3}\right), 3.41$ (br s, 1H, NH), $5.11(\mathrm{~s}, 1 \mathrm{H}, \mathrm{CH})$, 6.89-6.96 (m, 3H, Ar), 7.10-7.14 (m, 5H, Ar). ${ }^{13} \mathrm{C}$ NMR (75.5 MHz, DMSO- $\left.d_{6}\right): \delta 23.1\left(\mathrm{CH}_{3}\right), 53.0(\mathrm{CH}), 114.2(\mathrm{CH}$, Ar), 115.0 (C, Ar), 120.9 (CH, Ar), 128.3 (CH, Ar), 128.7 (CH, Ar), 130.1 (CH, Ar), 132.3 (CH, Ar), 147.4 (C-N, Ar),
$157.1(\mathrm{C}=\mathrm{N}), 162.8(\mathrm{C}-\mathrm{O}), 164.4(\mathrm{~S}-\mathrm{C}=\mathrm{N}), 176.9(\mathrm{C}=\mathrm{O})$. HRESIMS: $341.1233[\mathrm{M}+\mathrm{H}]^{+}$.

\subsubsection{4 | 5-Phenyl-2-(2-(1-(3-methoxy-4- hydroxyphenyl)ethylidene)hydrazinyl)thiazol- $4(5 H)$-one (16)}

Crystallization from toluene afforded white crystals, yield 69\%. M.p. $\left({ }^{\circ} \mathrm{C}\right): 174 \mathrm{IR}(\mathrm{KBr}): 3,411(\mathrm{~N}-\mathrm{H}), 1,710(\mathrm{C}=\mathrm{O})$, $1,605(\mathrm{C}=\mathrm{N}) \mathrm{cm}^{-1}$. ${ }^{1} \mathrm{H}$ NMR (300 MHz, DMSO- $\left.d_{6}\right): \delta 2.11$ $\left(\mathrm{CH}_{3}\right), 3.64$ (br s, $\left.1 \mathrm{H}, \mathrm{NH}\right), 4.33\left(\mathrm{OCH}_{3}\right), 5.00(\mathrm{~s}, 1 \mathrm{H}, \mathrm{CH})$, 6.90-7.03 (m, 3H, Ar), 7.10-7.16 (m, 5H, Ar). ${ }^{13} \mathrm{C}$ NMR $\left(75.5 \mathrm{MHz}, \mathrm{DMSO}-d_{6}\right): \delta 20.1\left(\mathrm{CH}_{3}\right), 52.1\left(\mathrm{OCH}_{3}\right), 53.0$ $(\mathrm{CH}), 114.2(\mathrm{CH}, \mathrm{Ar}), 115.0(\mathrm{C}, \mathrm{Ar}), 120.9(\mathrm{CH}, \mathrm{Ar}), 128.3$ (CH, Ar), $129.3(\mathrm{CH}, \mathrm{Ar}), 130.1(\mathrm{CH}, \mathrm{Ar}), 132.3(\mathrm{CH}, \mathrm{Ar})$, $137.4(\mathrm{C}-\mathrm{N}, \mathrm{Ar}), 157.1(\mathrm{C}=\mathrm{N}), 162.8(\mathrm{C}-\mathrm{O}), 164.4(\mathrm{~S}-$ $\mathrm{C}=\mathrm{N}), 176.9(\mathrm{C}=\mathrm{O})$. HRESIMS: $356.1162[\mathrm{M}+\mathrm{H}]^{+}$.

\subsubsection{5 | 5-Phenyl-2-(2-(1-(3,4- dimethylphenyl)ethylidene)hydrazinyl)thiazol- 4(5H)-one (17)}

Crystallization from toluene afforded white crystals, yield 73\%. M.p. $\left({ }^{\circ} \mathrm{C}\right): 181 \mathrm{IR}(\mathrm{KBr}): 3,410(\mathrm{~N}-\mathrm{H}), 1,710(\mathrm{C}=\mathrm{O})$, $1,605(\mathrm{C}=\mathrm{N}) \mathrm{cm}^{-1} \cdot{ }^{1} \mathrm{H}$ NMR $\left(300 \mathrm{MHz}, \mathrm{DMSO}-d_{6}\right): \delta$ $2.12\left(\mathrm{CH}_{3}\right), 2.18\left(\mathrm{CH}_{3}\right), 3.64$ (br s, $\left.1 \mathrm{H}, \mathrm{NH}\right), 5.03(\mathrm{~s}, 1 \mathrm{H}$, $\mathrm{CH}), 6.90-7.00(\mathrm{~m}, 3 \mathrm{H}, \mathrm{Ar}), 7.10-7.16(\mathrm{~m}, 5 \mathrm{H}, \mathrm{Ar}) .{ }^{13} \mathrm{C}$ NMR (75.5 MHz, DMSO- $\left.d_{6}\right): \delta 20.1\left(\mathrm{CH}_{3}\right), 52.1\left(\mathrm{OCH}_{3}\right)$, $53.0(\mathrm{CH}), 114.2(\mathrm{CH}, \mathrm{Ar}), 115.0(\mathrm{C}, \mathrm{Ar}), 120.9(\mathrm{CH}, \mathrm{Ar})$, $128.3(\mathrm{CH}, \mathrm{Ar}), 129.3(\mathrm{CH}, \mathrm{Ar}), 130.1(\mathrm{CH}, \mathrm{Ar}), 132.3(\mathrm{CH}$, Ar), $137.4(\mathrm{C}-\mathrm{N}, \mathrm{Ar}), 157.1(\mathrm{C}=\mathrm{N}), 162.8(\mathrm{C}-\mathrm{O}), 164.4$ $(\mathrm{S}-\mathrm{C}=\mathrm{N}), 176.9(\mathrm{C}=\mathrm{O})$. HRESIMS: $338.1244[\mathrm{M}+\mathrm{H}]^{+}$.

\section{4 | Physicochemical properties}

Four physicochemical properties, molecular weight, lipophilic index (octanol-water partitioning, log $\mathrm{P}$ ), hydrogen bond donors (H-bond donors) and hydrogen bond acceptors (H-bond acceptors), were calculated for thiazolidinones compounds using the Program MarvinSketch 16.7.18 (ChemAxon Ltd., Budapest). These descriptors were used to the application of Lipinski rules. The Lipinski's rule states that bioavailable molecules are more likely to have $<5 \mathrm{H}$-bond donors, $<10$ H-bond acceptors, $<500$ molecular weight and $<5 \mathrm{log}$ $P .{ }^{[22]}$ The Lipinski's index positivity is achieved to molecules that have no more than one violation (descriptor's values that is out of the range).

\section{5 | Animals}

Experiments were performed on male C57Bl/6 mice (18$22 \mathrm{~g}$ ) obtained from the Animal Facilities of Centro de Pesquisas Gonçalo Moniz/FIOCRUZ (Brazil). Animals were 
housed in temperature-controlled rooms $\left(22-25^{\circ} \mathrm{C}\right)$, under a 12:12 hr light-dark cycle, with access to water and food ad libitum. All behavioral tests were performed between 8:00 a.m. and 5:00 p.m., and animals were only tested once. Animal care and handling procedures were in accordance with the National Institutes of Health guide for the care and use of Laboratory animals (NIH, 8023) and the Institutional Animal Care and Use Committee FIOCRUZ (CPqGM 025/2011). Every effort was made to minimize the number of animals used and to avoid any discomfort. Behavioral tests were done without knowing to which experimental group each mouse belonged. Results shown are from two independent experiments performed.

\section{6 | Oxaliplatin-induced peripheral neuropathy and treatments}

Peripheral neuropathy was induced in mice by a single intraperitoneal (i.p.) administration of $3 \mathrm{mg} / \mathrm{kg}$ oxaliplatin (Glenmark, India) dissolved in 5\% dextrose at $100 \mu 1 .{ }^{[23]}$ Ten days after oxaliplatin injection, test compounds (thiazolidinones; 5-40 mg/kg), reference drug (gabapentin, $150 \mathrm{mg} /$ $\mathrm{kg}$, Pfizer, São Paulo, Brazil) or vehicle $(200 \mu \mathrm{l})$ was given by intraperitoneal route and the nociception was assessed 1 , 2, 4, 6 and $8 \mathrm{hr}$ after injection. The dose of gabapentin to induce the maximum effect was selected according to a previous study in mice. ${ }^{[24]}$ For the antagonism assay, the PPAR $\gamma$ antagonist GW9662 (Sigma-Aldrich, St. Louis, MO, USA) was used. GW9662 was dissolved in 4\% DMSO and immediately given as an intraperitoneal injection $(4 \mathrm{mg} / \mathrm{kg})$ to the mice $24 \mathrm{hr}$ prior to test compounds. ${ }^{[25]}$

\section{7 | Assessment of nociceptive threshold: von Frey filaments}

The threshold to mechanical stimulation was measured with von Frey filaments (Stoelting, Chicago, IL, USA). In a quiet room, mice were placed in acrylic cages $(12 \times 10 \times 17 \mathrm{~cm})$ with wire grid floor which allowed full access to the ventral aspect of the hind paws, 30 min before the beginning of the test. A logarithmic series of nine filaments were applied to the left hindpaw to determine the threshold stiffness required for $50 \%$ paw withdrawal according to the non-parametric method of Dixon, as described by Chaplan and collaborators. ${ }^{[26,27]}$ A positive response was characterized by the removal of the paw followed by clear flinching movements. The development of sensorial neuropathy was characterized by mechanical hypersensitivity, indicated by the paw withdrawal threshold reduction. The effects of thiazolidinones on the sensorial neuropathy were represented as paw withdrawal threshold and as percent maximum possible effect (\% of inhibition), considering the reversion of the mechanical hypersensitivity at 2-hr time-point.

\section{8 | Rota-rod test}

To evaluate possible non-specific muscle-relaxant or sedative effects of thiazolidinones, mice were submitted to the rota-rod test. The rota-rod apparatus (Insight, Ribeirão Preto, Brazil) consisted of a bar with a diameter of $3 \mathrm{~cm}$, subdivided into five compartments. The bar rotated at a constant speed of eight revolutions per min. The animals were selected $24 \mathrm{hr}$ previously by eliminating those mice that did not remain on the bar for two consecutive periods of $120 \mathrm{~s}$. Animals received intraperitoneal administration of diazepam $(10 \mathrm{mg} / \mathrm{kg}$, reference drug; Cristália, Itapira, Brazil), thiazolidinones $(40 \mathrm{mg} / \mathrm{kg}$ ), or vehicle, and $1 \mathrm{hr}$ afterward, were placed on a rotating rod. The resistance to falling was measured up to $120 \mathrm{~s}$. The results are expressed as the average time (s), and the animals remained on the rota-rod in each group.

\section{9 | Open-field test}

To assess the possible effects of thiazolidinones on locomotor activity, mice were evaluated in an open-field test. ${ }^{[28]}$ Mice were treated with diazepam $(10 \mathrm{mg} / \mathrm{kg}$; reference drug), thiazolidinones $(40 \mathrm{mg} / \mathrm{kg}$ ) or vehicle by intraperitoneal route and $1 \mathrm{hr}$ afterward were placed individually in a wooden box $(40 \times 60 \times 50 \mathrm{~cm})$ with the floor divided into 12 squares. The number of squares crossed with the four paws was counted for a period of $3 \mathrm{~min}$.

\subsection{0 | Data analysis}

All data are presented as means \pm standard error of the mean (SEM) of measurements made on six animals in each group. Von Frey data were analyzed using two-way ANOVA (group and time) followed by Bonferroni's multiple comparisons. Remaining data were analyzed using one-way ANOVA followed by Tukey's posttest. All data were analyzed using the Prism 5 computer software (GraphPad, San Diego, CA, USA). Statistical differences were considered to be significant at $p<.05$. In the present study, to determine the $\mathrm{ED}_{50}$ values of thiazolidinones, the compounds were administered at doses ranging between 5 and $40 \mathrm{mg} / \mathrm{kg}$. Four doses were used for each compound. $\mathrm{ED}_{50}$ values and confidence limits were calculated by computer-assisted log-probit analysis according to Litchfield and Wilcoxon. ${ }^{[29]} \mathrm{ED}_{50}$ values were calculated using the percent maximum possible effect, considering the inhibition of the mechanical hypersensitivity at 2-hr time-point.

\section{3 | RESULTS}

\section{1 | Chemistry}

The synthesis of 15 thiazolidinones (3-17) was accomplished in two steps, as outlined in Figure 1. The condensation 
<smiles>[R][I-]1=CC=CC(C(=C)C)=C1</smiles>

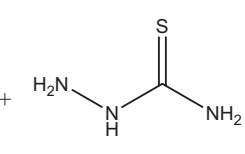

(1) (a)<smiles>[R][I-]c1ccc(/C(C)=N/NC(N)=S)cc1</smiles>

(2)

(b)

\begin{tabular}{|c|c|}
\hline$=\mathrm{H}(\mathbf{3})$ & $\mathrm{R}=4-\mathrm{Cl}(\mathbf{1 1})$ \\
\hline $4-\mathrm{CH}_{3}(\mathbf{4})$ & $4-\mathrm{CF}_{3}(\mathbf{1 2})$ \\
\hline $4-\mathrm{OCH}_{3}(\mathbf{5})$ & $3-\mathrm{CF}_{3}(13)$ \\
\hline 4- $\mathrm{C}\left(\mathrm{CH}_{3}\right)_{3}(\mathbf{6})$ & $3,4-\mathrm{OCH}_{3}(\mathbf{1 4})$ \\
\hline 4-Ph (7) & $3,4-\mathrm{OH}(\mathbf{1 5})$ \\
\hline $4-\mathrm{OH}(\mathbf{8})$ & $3-\mathrm{OCH}_{3}-4-\mathrm{OH}(\mathbf{1 6})$ \\
\hline $4-\mathrm{NO}_{2}(9)$ & $3,4-\mathrm{CH}_{3}(\mathbf{1 7})$ \\
\hline
\end{tabular}

4-F (10)
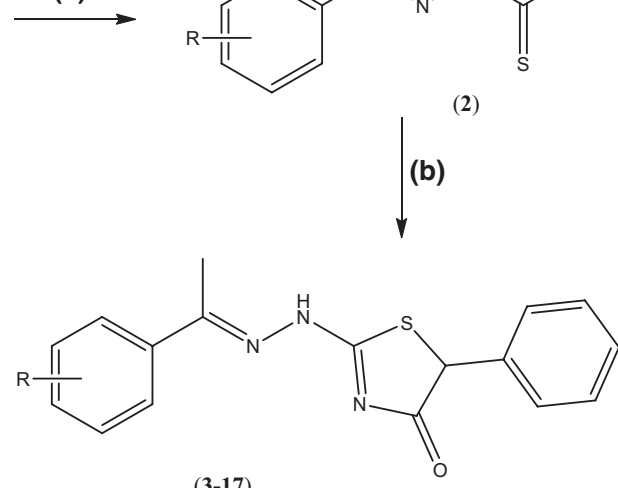

F IG URE 1 Synthesis of thiazolidinones (3-17). Reagents and conditions: (a) acetic acid (cat.), ethanol, ultrasound bath, $30 \mathrm{~min}$. (b) ethyl 2-chloro2-phenyl acetate, $\mathrm{NaOAc}$, ethanol, reflux, $10 \mathrm{hr}$ reaction between respective aryl ketone and thiosemicarbazide 1 afforded aryl thiosemicarbazones. Synthesized thiosemicarbazones $\mathbf{2}$ were then reacted in basic and reflux heating conditions with ethyl 2-chloro-2-phenyl acetate, forming 3-17 compounds. Thiazolidinones were isolated in acceptable yields $(41 \%-86 \%)$ and purity (>95\%). The structures were determined by ${ }^{1} \mathrm{H}$ and ${ }^{13} \mathrm{C}$ NMR, IR and mass spectra. An analysis of ${ }^{1} \mathrm{H}$ NMR for these compounds also revealed only one isomer. Chemical shifts in the hydrogen of iminic group are suggestive of an E-geometry. ${ }^{[21]}$ To study the structure-activity relationships, we firstly used monosubstituents attached at 4-position of phenyl ring $\left(\mathrm{CH}_{3}, \mathrm{~F}, \mathrm{Cl}\right.$, $\mathrm{Ph}, \mathrm{OCH}_{3}, \mathrm{CF}_{3}, \mathrm{OH}, \mathrm{NO}_{2}$ ). In these substituent series, the $\sigma \mathrm{p}$ Hammet values ranged from -0.37 for $\mathrm{OH}$ to +0.78 for $\mathrm{NO}_{2}$; therefore, the electronic contribution of the para-substituent on the phenyl group could vary significantly and allowing us to investigate the nature of each substituent for activity. Subsequently, we examined few substituents attached at 3-position or at both 3- and 4-positions, which allowed us to investigate the importance of position of a substituent for activity.

\section{2 | Pharmacological evaluation}

After structural characterization of thiazolidinones 3-17, the therapeutic potential of the new thiazolidinone compounds was evaluated in an established oxaliplatin-induced painful neuropathy model. Behavioral testing using von Frey, rotarod and open field were taken at baseline and at different times after treatment, and the antinociceptive activity was expressed as reduction in mechanical hypersensitivity. First, compounds were tested at single dose of $40 \mathrm{mg} / \mathrm{kg}$ by intraperitoneal route. This dose was established in a preliminary study of systemic toxicity showing that the designed thiazolidinones at $40 \mathrm{mg} / \mathrm{kg}$ did not affect the animal corporal mass and did not induce any variation in the general appearance or evident toxic signs in the treated mice. Following the initial test, the most active compounds without motor performance interference were selected to evaluation of dose-response relationship, efficacy and potency $\left(\mathrm{ED}_{50}\right)$. Gabapentin was used as the gold standard drug.

To monitor well-being, mice were observed and weighed daily throughout the experiment. All mice survived until the end of study. There were no signs of distress or general toxicity. Administration of oxaliplatin has been shown to induce neuropathy-associated behavioral mechanical hypersensitivity in mice without causing severe body weight loss or motor impairment (Figures 2 and 3). Behavioral signs of sensorial neuropathy were evident 3 days after the oxaliplatin treatment, peaked around 8 days persisting throughout the experimental time (12 days). To determine whether thiazolidinone compounds reduce the nociceptive hypersensitivity in chemotherapy-induced neuropathy, the paw withdrawal thresholds in neuropathic mice treated with thiazolidinone or vehicle were measured. All tested thiazolidinones showed statistically significant antinociception, but on a different scale of magnitude (Figure 2). Compounds 3, 4, 5, 6 and 11 exhibited discrete and short-lived antinociceptive effect. Compounds 7, 8, 9 and 10 showed a moderate antinociception. Compounds 12, 13, 14 and 17 showed improved antinociception, while $\mathbf{1 5}$ and $\mathbf{1 6}$ presented maximum effect and long-lasting antinociception.

To rule out relaxing or motor impairing effects, the influence of the most active compounds 12, 14, 15, 16 on the motor performance was evaluated (Figure 3). Administration of thiazolidinones 12, 15 as well as $\mathbf{1 6}$ at therapeutic doses (40 mg/ $\mathrm{kg}$ ) did not affect the motor performance of the mice, as tested in the rota-rod (Figure 3a) and open-field (Figure 3b) tests. As expected, $1 \mathrm{hr}$ of treatment with the central nervous system depressant diazepam (10 mg/kg, ip), a standard drug, reduced the time of mice on the rota-rod and the number of crossings on the open field. Similarly, compound 14 (40 mg/kg i.p.) caused motor impairment.

Taking impairing of motor function and magnitude of effect as parameters steering, compounds 12, 15 and 16 were further investigated. The physicochemical properties of 

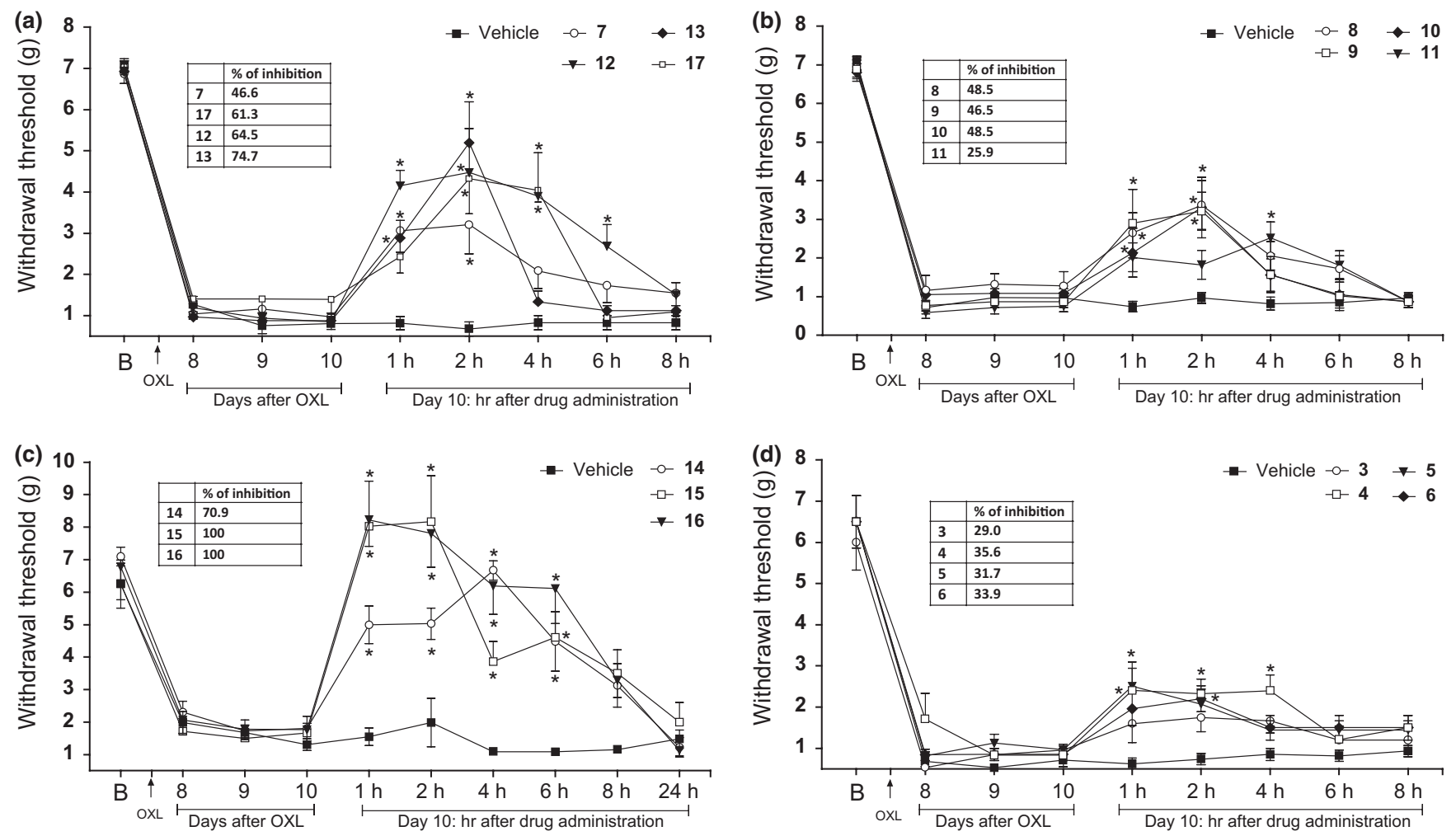

F I G U RE 2 Effects of thiazolidinones on oxaliplatin-induced nociceptive hypersensitivity in mice. Mechanical nociceptive threshold was tested 10 days after the oxaliplatin $(3 \mathrm{mg} / \mathrm{kg}$ ) treatment. Mice were treated with thiazolidinones ( $40 \mathrm{mg} / \mathrm{kg}$ ) or vehicle (200 $\mu \mathrm{l}$; control group) by intraperitoneal route (zero time), and the mechanical thresholds were evaluated at different times after injection, as indicated in the figure. Mechanical thresholds are represented as 50\% paw withdrawal threshold, in grams. Panel a: antinociceptive effects of 7, 12, 13 and 17. Panel b: antinociceptive effects of 8, 9, 10 and 11. Panel c: antinociceptive effects of 14, 15 and 16. Panel d: antinociceptive effects of 3, 4, 5 and 6. Inset on panels showing the percent maximum possible effect (\% of inhibition), considering the mechanical hypersensitivity inhibition at 2-hr point. Data are expressed as means $\pm \mathrm{SEM} ; n=6$ mice per group. *Significantly different from vehicle-treated group $(p<.05)$, as determined by two-way ANOVA followed by the Bonferroni's test
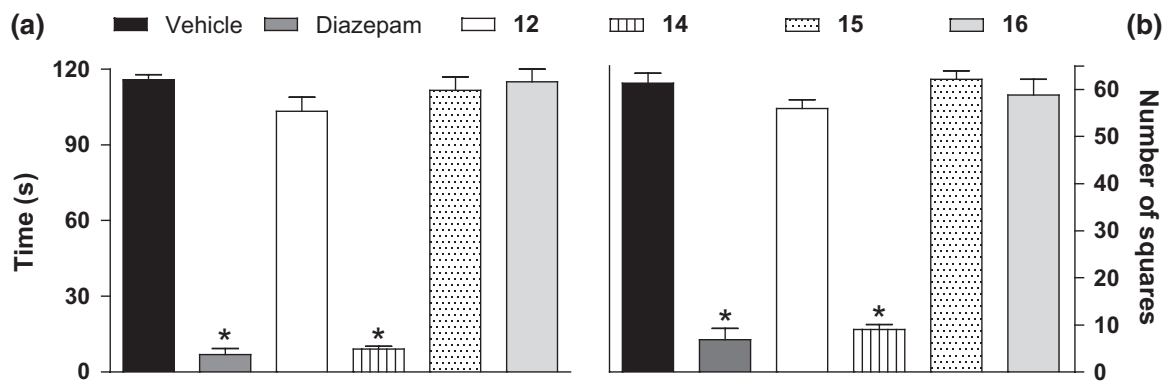

F I G U R E 3 Effects of thiazolidinones on motor function. Bar graphs representing (a) the run time on the rota-rod and (b) the number of square crossings in the open-field test, $1 \mathrm{hr}$ after the intraperitoneal administration of thiazolidinones $\mathbf{1 2}, \mathbf{1 4}, \mathbf{1 5} \mathrm{and} \mathbf{1 6}(40 \mathrm{mg} / \mathrm{kg}), \mathrm{vehicle}(200 \mu \mathrm{l}$; control group) or diazepam $(10 \mathrm{mg} / \mathrm{kg}$; reference drug). Data are reported as means $\pm \mathrm{SEM} ; n=6$ mice per group. *Significantly different from the vehicle-treated group $(p<.001)$, as determined by ANOVA followed by the Tukey's range test

12, 15 and 16 were analyzed by testing the Lipinski rules. According to Table 1, thiazolidinones $\mathbf{1 5}$ and $\mathbf{1 6}$ satisfied all Lipinski rules, and thiazolidinone $\mathbf{1 2}$ satisfied three of the four Lipinski criteria. Next, pharmacological properties of 12, 15 and 16 were investigated and compared to gabapentin pharmacological profile (Figure 4). Thiazolidinones $\mathbf{1 2}$ and $\mathbf{1 5}$ display a dose-related antinociceptive effect, with similar pharmacological efficacy compared to gabapentin. Thiazolidinone 12 exhibited an $\mathrm{ED}_{50}$ value of $18.6 \mathrm{mg} / \mathrm{kg}$ to reverse mechanical hypersensitivity, indicating a similar potency relative to gabapentin $\left(\mathrm{ED}_{50}\right.$ value of $21.3 \mathrm{mg} / \mathrm{kg}$ ). In contrast, compounds $\mathbf{1 5}$ and $\mathbf{1 6}$ were 6- and 12-fold more potent that gabapentin, respectively, displaying $\mathrm{ED}_{50}$ values of 3.3 and $1.7 \mathrm{mg} / \mathrm{kg}$. The dose-related antinociceptive effect 


\begin{tabular}{llllll} 
Compound & $\begin{array}{l}\text { Molecular } \\
\text { weight }^{\mathbf{a}}(\mathbf{g} / \mathbf{m o l})\end{array}$ & $\mathbf{L o g}^{\mathbf{b}}$ & $\begin{array}{l}\text { H-bond } \\
\text { acceptors }^{\mathbf{c}}\end{array}$ & $\begin{array}{l}\text { H-bond } \\
\text { donors }^{\mathbf{d}}\end{array}$ & $\begin{array}{l}\text { Lipinski's } \\
\text { rule }\end{array}$ \\
\hline $\mathbf{1 2}$ & 377 & 5.02 & 8 & 1 & YES \\
$\mathbf{1 5}$ & 341 & 3.56 & 7 & 3 & YES \\
$\mathbf{1 6}$ & 355 & 3.60 & 7 & 2 & YES \\
\hline
\end{tabular}

T A B L E 1 Lipinski rules parameters results for the antinociceptive thiazolidinone compounds

${ }^{\text {a }}$ The upper limit of the molecular weight is 500

${ }^{\mathrm{b}}$ The $\log \mathrm{P}$ value should not be $>5$.

${ }^{\mathrm{c}}$ The upper limit of the number of hydrogen bond acceptors is 10 .

${ }^{\mathrm{d}}$ The upper limit of the number of hydrogen bond donors is 5 . Number of violation allowed is 1 .

of thiazolidinone $\mathbf{1 6}$ was long-lasting and with an enhanced efficacy than that of gabapentin. Aiming to confirm the contribution of PPAR activation to the antinociceptive effects of the designed thiazolidinones, its effects on the mechanical hypersensitivity of neuropathic mice were also evaluated in the presence of GW9662, a PPAR $\gamma$ antagonist. Systemic pretreatment with GW9662 (4 mg/kg ip) completely prevented the antinociceptive effect of $\mathbf{1 2}$ and 15, and reduced in 66\% the 16-induced antinociception (Figure 4d).

\section{4 | DISCUSSION}

In the present study, the analgesic potential of the designed thiazolidinones was analyzed in a comparative way, by varying the substituents attached to the phenyl ring. First, the unsubstituted thiazolidinone $\mathbf{3}$ was evaluated, showing low antinociceptive activity on the chemotherapy-induced neuropathy model. Similar to $\mathbf{3}$, the substituted thiazolidinone presenting a methyl $\mathbf{4}$, methoxy 5 or butyl $\mathbf{6}$ groups also presented low activity. These results indicated that the attachment of apolar groups does not increase antinociceptive activity in thiazolidinone. The substituted thiazolidinone containing a phenyl group 7 displayed slightly superior activity than unsubstituted thiazolidinone $\mathbf{3}$. Similarly, when a polar group, such as hydroxyl $\mathbf{8}$ or nitro $\mathbf{9}$, was attached in the structure of thiazolidinone, the antinociceptive activity was increased up to $48 \%$ of mechanical hypersensitivity reversion, as observed in compound $\mathbf{8}$. Compounds containing halogen atoms were also evaluated. The thiazolidinone containing fluoro 10, but not chloro 11, presented improved antinociceptive activity than unsubstituted thiazolidinone $\mathbf{3}$. In addition, the trifluoromethyl $\mathbf{1 2}$ and $\mathbf{1 3}$ enhanced more than twofold the antinociceptive activity of the unsubstituted thiazolidinone 3. An important improvement of activity was observed when two substituents were attached, such as 3,4-dimethoxy, 3,4-dihydroxyl and 3-methoxy-4-hydroxy groups, yielding compounds 14, 15, 16, respectively. However, compound presenting two methyl groups $\mathbf{1 7}$ was slightly less active than other bi-substituent compounds. In summary, the identification of new synthetic antinociceptive compounds was achieved here by using the thiazolidinone heterocyclic as main structural pharmacophoric group. By varying the substituents attached to the phenyl ring, it was possible to observe substituents that maintain or increase the antinociceptive activity in comparison with the unsubstituted thiazolidinone.

Most tests that are used to study pain in animals involve motor responses to nociceptive stimuli. ${ }^{[30]}$ Therefore, the reduction in the behavioral nociceptive response induced by new substances could be associated with motor deficits rather than antinociceptive activity. To avoid misinterpretation of data, the motor performance of mice treated with thiazolidinones was evaluated. The negative results of thiazolidinones 12, 15 and $\mathbf{1 6}$ on the motor assays corroborate their antinociceptive properties indicated by the nociceptive test. Considering that thiazolidinones 12, 15 and $\mathbf{1 6}$ display antinociceptive activity without impairing general motor, their pharmacological characterization was next performed through the evaluation of dose-response relationship, efficacy and potency $\left(\mathrm{ED}_{50}\right)$. In addition, physicochemical properties of these thiazolidinones were calculated, allowing the application of the Lipinski rules to predict the bioavailable of this compounds. ${ }^{[22]}$

Despite advances in pharmaceutical sciences, drug discovery is still an expensive and laborious process. An obstacle to be transposed is the difficulty of identifying bioactive compounds with the appropriate properties that are ultimately successful in the clinical phase. A frequent problem related to the risk of clinical failure of new drugs is low oral bioavailability of the compounds. Numerous studies have connected the bioavailability to the physiochemical properties of molecules. ${ }^{[31]}$ Among the different approaches that have been used to assess the properties of molecules in early stages of the discovery-research process, the Lipinski rules are the most widely employed. This set of rules identify descriptors based on intrinsic and physicochemical properties that help to infer whether the studied compounds might have, or not, problems regarding oral bioavailability. ${ }^{[22]}$ In the present study, the physicochemical properties of the thiazolidinone compounds with the improved antinociceptive activity were analyzed by testing the Lipinski rules. Compounds $\mathbf{1 5}$ and $\mathbf{1 6}$ satisfied all Lipinski rules, while 12 satisfied three of the four Lipinski criteria. Compounds showing physicochemical descriptors 

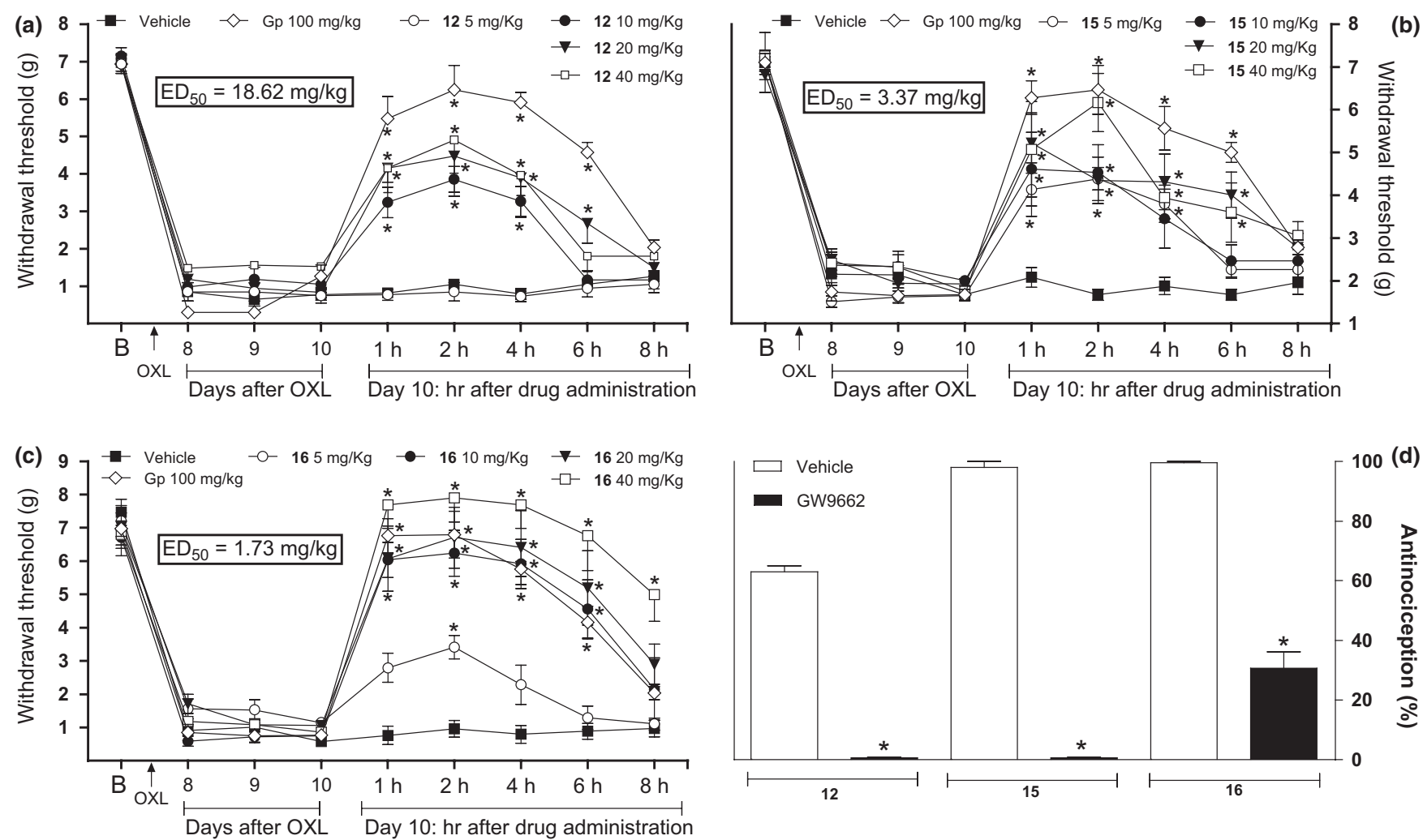

F I G U R E 4 Dose-response curves of thiazolidinones on oxaliplatin-induced nociceptive hypersensitivity in mice. Mechanical nociceptive threshold was tested 10 days after the oxaliplatin $(3 \mathrm{mg} / \mathrm{kg})$ treatment. Mice were treated with thiazolidinones $(5-40 \mathrm{mg} / \mathrm{kg})$ or vehicle $(200 \mu \mathrm{l}$; control group) by intraperitoneal route (zero time), and the mechanical thresholds were evaluated at different times after injection, as indicated in the figure. Mechanical thresholds are represented as 50\% paw withdrawal threshold, in grams. Panels A, B and C show the dose-response curves of 12, 15 and 16, respectively. Gabapentin $(100 \mathrm{mg} / \mathrm{kg} / \mathrm{ip})$ was used as reference drug. Inset on panels showing the $\mathrm{ED}_{50} \mathrm{values}$ Panel $\mathrm{D}$ shows data from PPAR antagonism assay. Bars representing the percent maximum possible antinociceptive effect, considering the mechanical hypersensitivity inhibition at 2-hr point. GW9662 (4 mg/kg; PPAR antagonist) or vehicle was administered by intraperitoneal route 24 hr prior to thiazolidinones 12 , 15 or 16. Data are expressed as means \pm SEM; $n=6$ mice per group. *Significantly different from vehicle-treated group $(p<.05)$, as determined by two-way ANOVA followed by the Bonferroni's test

values within an established range are more likely to be membrane permeable and more easily absorbed by the body. ${ }^{[31]}$ Based on this analysis, $\mathbf{1 5}$ and $\mathbf{1 6}$ are predicted to have suitable bioavailability after oral administration, increasing the pharmaceutical potential of these molecules.

From the dose-response studies, it was possible to demonstrate that 12, 15 and $\mathbf{1 6}$ display a dose-related antinociceptive effect and improved pharmacological properties. The antinociceptive effect of $\mathbf{1 6}$ was more potent, long-lasting and with an enhanced efficacy than gabapentin, the gold standard drug used for clinical control of neuropathic pain, which is an ideal profile for drug development of novel pain killers. It is important to note that the antinociceptive effects of 12, 15 and 16 were prevented by the PPAR $\gamma$ antagonist, suggesting that these compounds have agonistic properties in PPAR $\gamma$. In fact, the potential of thiazolidinones as a new therapeutic approach for oxaliplatin-induced neuropathic pain has been demonstrated. ${ }^{[20]}$ This concept is based on the causal relationship between oxaliplatin-induced neuropathic pain and oxidative stress. ${ }^{[32]}$ Mitochondria and peroxisomes, the main organelles involved with the cellular redox balance, have been considered key targets of oxaliplatin neurotoxicity. ${ }^{[33]}$ Oxaliplatin-evoked painful neuropathy is associated with impaired mitochondrial function in peripheral nerve axons, resulting in a chronic axonal energy deficit that may be the cause of the neuropathy symptoms. ${ }^{[33,34]}$ In addition, a relationship between neuropathy and catalase impairment has been demonstrated. ${ }^{[35,36]}$ Catalase, an important antioxidant defense enzyme, shows decreased efficiency in pain pathways during oxaliplatin-induced painful neuropathy. ${ }^{[20]}$ The activity and gene expression of this enzyme are increased by PPAR $\gamma$ activation, triggering antioxidant mechanisms. ${ }^{[37]}$ In fact, activation of PPAR in the pain pathways protects neuronal damage induced by oxidative stress. ${ }^{[17,18,38]}$ PPAR is a class of ligand-activated transcription factors of the nuclear receptor superfamily. In addition to their physiological role as a regulator of lipid metabolism, PPAR ligands repress the neuroinflammatory response in neurodegenerative conditions, by molecular mechanisms of gene transrepression. ${ }^{[39]}$ The ability of these nuclear receptors to reduce 
neuroinflammation and protect against neuronal oxidative damage makes them attractive targets for intervention in neurological diseases, such as neurodegenerative diseases, spinal cord injury and neuropathic pain. ${ }^{[16,39-42]}$ Data showed in the present study are in line with this propose, reinforcing that thiazolidinones are strong antinociceptive compounds, able to substantially reverse the nociceptive sensitization associated with oxaliplatin-induced neuropathy.

In conclusion, the present results show that the structural design and synthesis of new thiazolidinones are an efficient medicinal chemistry approach to the identification of new compounds with improved pharmacological properties and potentially useful to treat oxaliplatin-induced neuropathic pain.

\section{ACKNOWLEDGEMENTS}

The authors wish to thank Central Analítica da UFPE for technical assistance in NMR and IR analyses. This work was supported by FAPESB [grant numbers PNX 0009/2009; RED0010/2012].

\section{CONFLICT OF INTEREST}

The authors declare that there are no conflicts of interest.

\section{REFERENCES}

[1] M. Haanpää, N. Attal, M. Backonja, R. Baron, M. Bennett, D. Bouhassira, G. Cruccu, P. Hansson, J. A. Haythornthwaite, G. D. Iannetti, T. S. Jensen, T. Kauppila, T. J. Nurmikko, A. S. Rice, M. Rowbotham, J. Serra, C. Sommer, B. H. Smith, R. D. Treede, Pain 2011, 152, 14.

[2] R. C. Jones, M. M. Backonja, Curr. Pain Headache Rep. 2013, 17, 363.

[3] A. B. O'Connor, Pharmacoeconomics 2009, 27, 95.

[4] S. Wolf, D. Barton, L. Kottschade, A. Grothey, C. L. Loprinzi, Eur. J. Cancer 2008, 44, 1507.

[5] B. N. Reeves, S. R. Dakhil, J. A. Sloan, S. L. Wolf, K. N. Burger, A. Kamal, N. A. Le-Lindqwister, G. S. Soori, A. J. Jaslowski, J. Kelaghan, P. J. Novotny, D. H. Lachance, C. L. Loprinzi, Cancer 2012, 118, 5171.

[6] W. Grisold, G. Cavaletti, A. J. Windebank, Neuro Oncol. 2012, 14,45 .

[7] A. Ibrahim, S. Hirschfeld, M. H. Cohen, D. J. Griebel, G. A. Williams, R. Pazdur, Oncologist 2004, 9, 8

[8] J. W. Albers, V. Chaudhry, G. Cavaletti, R. C. Donehower, Cochrane Database Syst. Rev. 2014, 3, CD005228.

[9] M. Ahmadian, J. M. Suh, N. Hah, C. Liddle, A. R. Atkins, M. Downes, R. M. Evans, Nat. Med. 2013, 19, 557.

[10] D. Della-Morte, R. Palmirotta, A. K. Rehni, D. Pastore, B. Capuani, F. Pacifici, M. L. De Marchis, K. R. Dave, A. Bellia, G. Fogliame, P. Ferroni, G. Donadel, F. Cacciatore, P. Abete, C. Dong, A. Pileggi, M. Roselli, C. Ricordi, P. Sbraccia, F. Guadagni, T. Rundek, D. Lauro, Pharmacogenomics 2014, 15, 2063.
[11] V. S. Jain, D. K. Vora, C. S. Ramaa, Bioorg. Med. Chem. 2013, $21,1599$.

[12] A. A. Amato, S. Rajagopalan, J. Z. Lin, B. M. Carvalho, A. C. Figueira, J. Lu, S. D. Ayers, M. Mottin, R. L. Silveira, P. C. Souza, R. H. Mourão, M. J. Saad, M. Togashi, L. A. Simeoni, D. S. Abdalla, M. S. Skaf, I. Polikparpov, M. C. Lima, S. L. Galdino, R. G. Brennan, J. D. Baxter, I. R. Pitta, P. Webb, K. J. Phillips, F. A. Neves, J. Biol. Chem. 2012, 287, 28169.

[13] K. P. Barbosa, L. A. Santos, E. L. Ribeiro, I. T. Fragoso, S. W. Rocha, A. K. Nunes, M. E. França, B. S. Silva, A. K. Silva, M. A. Donato, F. O. Gomes, T. G. Silva, I. R. Pitta, M. R. Pitta, M. C. Lima, F. D. Uchôa, S. L. Galdino, C. A. Peixoto, Eur. J. Pharmacol. 2013, 718, 197.

[14] J. R. R. LoVerme, G. La Rana, J. Fu, J. Farthing, G. Mattace-Raso, R. Meli, A. Hohmann, A. Calignano, D. Piomelli, J. Pharmacol. Exp. Ther. 2006, 319, 1051.

[15] B. K. Taylor, N. Dadia, C. B. Yang, S. Krishnan, M. Badr, Inflammation 2002, 26, 121.

[16] S. W. Park, J. H. Yi, G. Miranpuri, I. Satriotomo, K. Bowen, D. K. Resnick, R. Vemuganti, J. Pharmacol. Exp. Ther. 2007, 320, 1002.

[17] S. B. Churi, O. S. Abdel-Aleem, K. K. Tumber, H. Scuderi-Porter, B. K. Taylor, J. Pain 2008, 9, 639.

[18] T. Maeda, N. Kiguchi, Y. Kobayashi, M. Ozaki, S. Kishioka, J. Pharmacol. Sci. 2008, 108, 341.

[19] J. Morgenweck, R. B. Griggs, R. R. Donahue, J. E. Zadina, B. K. Taylor, Neuropharmacology 2013, 70, 236e246.

[20] M. Zanardelli, L. Micheli, L. Cinci, P. Failli, C. Ghelardini, L. Di Cesare Mannelli, PLOS ONE 2014, 9, e102758.

[21] D. C. Greenbaum, Z. Mackey, E. Hansell, P. Doyle, J. Gut, C. R. Caffrey, J. Lehrman, P. J. Rosenthal, J. H. McKerrow, K. Chibale, J. Med. Chem. 2004, 47, 3212.

[22] C. A. Lipinski, F. Lombardo, B. W. Dominy, P. J. Feeney, $A d v$. Drug Deliv. Rev. 2001, 46, 3 .

[23] P. Gauchan, T. Andoh, K. Ikeda, M. Fujita, A. Sasaki, A. Kato, Y. Kuraishi, Biol. Pharm. Bull. 2009, 32, 732.

[24] N. Kusunose, S. Koyanagi, K. Hamamura, N. Matsunaga, M. Yoshida, T. Uchida, M. Tsuda, K. Inoue, S. Ohdo, Mol. Pain. 2010, 26, 83.

[25] T. Huang, D. Gao, Y. Hei, X. Zhang, X. Chen, Z. Fei, Brain Res. 2016, 1642, 478 .

[26] S. R. Chaplan, F. W. Bach, J. W. Pogrel, J. W. Chung, T. L. Yaksh, J. Neurosci. Methods 1994, 53, 55.

[27] W. J. Dixon, Annu. Rev. Pharmacol. Toxicol. 1980, $20,441$.

[28] A. L. S. Rodrigues, G. L. Silva, A. S. Mateussi, E. S. Fernandes, O. G. Miguel, R. A. Yunes, J. B. Calixto, A. R. S. Santos, Life Sci. 2002, 70, 1347.

[29] J. T. Litchfield Jr, F. A. Wilcoxon, J. Pharmacol. Exp. Ther. 1949, 96, 99.

[30] D. Le Bars, M. Gozariu, S. W. Cadden, Pharmacol. Rev. 2001, 53, 597.

[31] P. Leeson, Nature 2012, 481, 455.

[32] L. Di Cesare Mannelli, M. Zanardelli, P. Failli, C. Ghelardini, J. Pain 2012, 13, 276.

[33] H. Zheng, W. H. Xiao, G. J. Bennett, Exp. Neurol. 2011, 232, 154.

[34] W. H. Xiao, G. J. Bennett, Pain 2012, 153, 704.

[35] S. S. Kamboj, R. K. Vasishta, R. Sandir, J. Neurochem. 2010, 112, 77.

[36] A. Masoud, R. Sandhir, Hum. Exp. Toxicol. 2012, 31, 1214.

[37] G. D. Girnun, F. E. Domann, S. A. Moore, M. E. Robbins, Mol. Endocrinol. 2002, 16, 2793. 
[38] E. Gray, M. Ginty, K. Kemp, N. Scolding, A. Wilkings, J. Neuroinflammation 2012, 9, 63.

[39] T. Maeda, S. Kishioka, Int. Rev. Neurobiol. 2009, 85, 165.

[40] M. T. Heneka, G. E. Landreth, M. Hüll, Nat. Clin. Pract. Neurol. 2007, 3, 496.

[41] G. Landreth, Q. Jiang, S. Mandrekar, M. Heneka, Neurotherapeutics 2008, 5, 481.

[42] C. R. Swanson, V. Joers, V. Bondarenko, K. Brunner, H. A. Simmons, T. E. Ziegler, J. W. Kemnitz, J. A. Johnson, M. E. Emborg, J. Neuroinflammation 2011, 8, 91.
How to cite this article: Moreira DRM, Santos DS, Santo RFE, et al. Structural improvement of new thiazolidinones compounds with antinociceptive activity in experimental chemotherapy-induced painful neuropathy. Chem Biol Drug Des. 2017;90:297-307. https://doi.org/10.1111/cbdd.12951 